\title{
COVID-19 with Pregnancy: Obstetricians should be Alert for Postpartum Exacerbation
}

\author{
Ahmed M Abbas ${ }^{1,2 *}$, Shimaa Salah Ali, ${ }^{2,3}$, Alya O Mohamed ${ }^{2,3}$, Alaa Rashad Ali, ${ }^{2,3}$, AlBatool \\ M AlMahdy ${ }^{2,3}$, Asmaa AboBakr ${ }^{2,3}$ and Shorouk M Adel ${ }^{2,3}$ \\ ${ }^{1}$ Department of Obstetrics \& Gynecology, Faculty of Medicine, Assiut University, Egypt \\ ${ }^{2}$ COvid-19 Research of Assiut University Association (CORAUNA) group \\ ${ }^{3}$ House-officer, Faculty of Medicine, Assiut University, Egypt
}

\begin{abstract}
Introduction: Physiological changes in pregnancy make them more susceptible to infection. SARS-CoV-2 infection ranges in severity from common cold to acute respiratory distress syndrome. With the incredible increase in numbers of coronavirus disease-2019 (COVID-19), multiple studies were done with the significant increase of COVID-19 infected pregnant women. These studies support that pregnant women are not only susceptible to infection but more liable for severity and complications that may end unfortunately by death.

Methods: In this review, we discuss the mode and steps of delivery in that situation. There is an argument about fetal transmission, possible methods of transmission, and recorded fetal complications. Breastfeeding is not a source of infection. The separation between the infected mother and her baby is under debate regarding many considerations taken.
\end{abstract}

Keywords

Pregnancy, Postpartum, COVID-19, Exacerbation

\section{Introduction}

In December 2019, an emerging disease of coronaviridae family (SARS-CoV-2) appeared in Wuhan, China, and spread globally in an incredible way leading to a pandemic that affects all populations, including pregnant women [1]. Data available about pregnancy is limited. Pregnant women and neonates are considered high-risk populations that need intensive care as it can increase morbidity and mortality rates.

Females are susceptible to infections during pregnancy in general as physiological changes during pregnancy in respiratory, endocrinal, immunological systems increase the sensitivity and severity of pneumonia [2]. This is attributed to the moving up of the diaphragm and relaxation of the chest ligaments, so the lung compliance and functional residual capacity decrease. Therefore, women compensate by hyperventilation that leads to more air inhalation and more risk to get the virus infection. Changes in the nasal mucosa by progesterone effect make the virus adherent to the mucosa and challenging to clear it. Besides, the decreased number of $T$ cells and their function during pregnancy, increase ACE2 receptors expression during pregnancy could increase the susceptibility for catching coronavirus disease-2019 (COVID-19) [3].
There are reported cases about severe COVID-19 during pregnancy and the postpartum stage. In Sweden, 53 women confirmed to be infected with COVID-19 aged between 2045 years old admitted to the intensive care unit (ICU); 13 of them are pregnant or postpartum less than one week [4]. In another study, 31 pregnant women with COVID-19 were reported, two women of them died after delivery due to respiratory complications [5]. Another case reported in Iran about a pregnant woman with respiratory deterioration in pregnancy needs ICU and intubation then termination of pregnancy. Finally, she died on the seventh day after delivery [6]. However, all these data considered not enough to prove COVID-19 severity in pregnancy and postpartum stage due

*Corresponding author: Dr. Ahmed M Abbas, MD, Department of Obstetrics and Gynecology, Assiut University, Women Health Hospital, 71511, Assiut Egypt, Egypt; Tel: +208-8241-4616, Fax: +208-8 920-2503.

Accepted: December 09, 2021

Published online: December 11, 2021

Citation: Abbas AM, Ali SS, Mohamed AO, et al. (2021) COVID-19 with Pregnancy: Obstetricians should be Alert for Postpartum Exacerbation. Annals Gynecol Obstet 5(1):138-141 
Citation: Abbas AM, Ali SS, Mohamed AO, et al. (2021) COVID-19 with Pregnancy: Obstetricians should be Alert for Postpartum Exacerbation. Annals Gynecol Obstet 5(1):138-141

to a limited number of studies up till now. In contrast to SARS and MERS, which have evidence of severe morbidity and mortality during pregnancy compared to the general population [7].

\section{Labor and Postpartum care}

Several studies have been recently released discussing the possible management and the special considerations that the obstetricians should adhere to when dealing with COVID-19 patients. During labor and postpartum care, obstetricians must take the necessary precautions before dealing with a suspected or a confirmed case. Patient encounters are classified into low, moderate, and high risk, and accordingly, the healthcare workers should take precautions [8].

Low-risk encounters, such as brief encounters with two meters distance between patient and healthcare giver, require standard precautions and surgical masks only. Obstetric examination, Ultrasonography, and vaginal or cesarean delivery are considered moderate risk encounters. Personal protective equipment (PPE) for such situations consists of a surgical cap, gloves, face shield or goggles, gown, and surgical mask or N95 respirator [8]. Patients that require cardiopulmonary resuscitation (CPR), endotracheal intubation, and use of supplemental oxygen in labor: nasal cannula, face mask, or non-rebreather mask are considered high risk and full PPE and precautions should be taken [8].

Till now, there is no sufficient evidence suggesting vertical transmission of SARS-COV-2, and therefore vaginal delivery is not contraindicated. However, it is obligatory to take the necessary precautions for both the mother and the obstetricians. Full PPE and air precautions should be taken, and delivery, whether vaginal or cesarean, should be performed in a negative air pressure room [9]. The decision of mode of delivery is made based on clinical and obstetric factors. Here are some guidelines for postnatal management:

If the patient is suspected of having COVID-19 but has no symptoms, a negative air pressure room is not required, and delivery can be performed in a regular room. The patient is then monitored and observed for ten days for any symptoms or signs of COVID-19. Otherwise, if a suspected case has any symptom or sign, then the delivery should be performed in a negative pressure ventilation room.

Postnatal management of suspected cases necessitates close monitoring of vital signs and symptoms to detect the development of any. If so, the patient should be moved to a negative pressure room.

Breastfeeding is not permissible until the mother has recovered completely or COVID-19 infection is ruled out. The neonate should also be completely isolated from the mother to reduce the risk of infection transmission [9].

On the occasion of a confirmed case of COVID-19, the patient should be immediately placed in a negative ventilation room where delivery should be placed, and all precautions for air transmission must be taken by all healthcare workers. Early clamping and cleaning of the newborn are encouraged to reduce the risk of infection transmission. No breastfeeding and total isolation of mother and neonate until complete recovery from COVID-19 is confirmed. Patient discharge is decided upon when the patient fulfills the discharge criteria:

1) Normal body temperature for more than three days,

2) Improvement of respiratory symptoms,

3) Chest X-ray reveals improvement of acute lesions,

4) Two consecutive negative nasopharyngeal swabs for SARSCOV-2 with more than 24 hours between every sample [10].

A case study in Wuhan, China, reported that seven pregnant female patients with SARS-COV-2 had good recoveries and did not require ICU admission throughout their hospital stay [10]. Another review showed that pregnant females with SARS-COV-2 are more liable to have adverse outcomes, as preeclampsia and preterm births, and premature rupture of membranes than non-pregnant females [11].

\section{Fetal complications}

Several studies state that there is no proof of vertical transmission of COVID-19 from a mother to a fetus and that neonates that were COVID-19 positive were positive due to horizontal transmission after birth [12]. The neonates born to COVID-19 positive mothers that tested negative showed no evidence of viral isolates in the amniotic fluid, cord blood, breast milk, and throat swabs of the neonates [8]. However, the majority of these neonates were born to mothers that contracted COVID-19 during the third trimester. Thus there is not enough data that can be postulated regarding the infection and its transmission if it occurs in early pregnancy. However, there is still a possibility that there might be vertical transmission from a mother to a child since two neonates that were born to positive mothers were tested positive shortly after their delivery. However, this possibility still needs further study [8].

The fetal complications of COVID-19 that have been recorded so far include, but are not limited to, miscarriage or spontaneous abortion, stillbirth, fetal distress, respiratory distress, preterm birth, fetal growth restriction (FGR), premature rupture of the fetal membrane, thrombocytopenia associated with abnormal liver function, and neonatal death $[8,13]$. Preterm birth may be the most common fetal complication [11].

\section{Effect on breastfeeding}

As known, breastfeeding is essential for both mother and infant health and has a positive economic and social effect for the whole family [14]. However, in the current situation concerning COVID-19, it is recommended to separate between mother and infant. It has been proved that breast milk is not considered as a route of transmission of COVID-19 from mother to infant as other known respiratory viral infections. Some authors going with the idea of separation, ignoring its importance, and without any evidence, others recommend keeping breastfeeding and promote infection control measures [15]. Some recommend using formula or donor breast milk rather than breastfeeding and direct contact between mother and child without sound reasons. 
Women suspected, or confirmed to have COVID-19 can keep breastfeeding and direct contact with her child taking in consideration the infection control measures as far as the mother is not severely affected as recommended by World Health Organization, Royal College of Obstetricians and Gynecologists and International Society of Ultrasound in Obstetrics and Gynecology (ISUOG) [16,17]. According to the CDC (Centers of Disease Control and Prevention), if mother tests are positive for COVID-19, the separation between the mother and child should be the first option, and the risks and benefits should be explained to the family. But if the mother and child are managed together, measures of infection control should be put in place such as, avoid kissing, put the mask, wash hands and protect him from cough and any respiratory secretions [18].

The separation helps to decrease the risk of disease transmission, but if the health of mother and child is the primary goal, there is some consideration that should be taken:

- Separation at the hospital may just delay the exposure of the child, but not prevent the infection as some families cannot keep that separation at home, and the exposure occurs sonar or later.

- Skin to skin contact between mother and infant improve infant health. It makes him more stable, increases blood glucose level, decreases heart rate, and respiratory rate [19].

- It also maintains thermoregulation, decreases the risk of jaundice, encourages bonding between mother and child, decreases the stress of birth, and increases the duration of breastfeeding, which is a good source of immunity to the infant.

- The separation stresses the mother and worsens the disease course.

- At home, the mother continue breastfeeding or express breast milk according to her health condition, telephone consultation of a pediatrician for assessment of the child and guide parents for any advice

- The infant needs check-up at 14 days after discharge by pharyngeal swab and stops the follow up after 28 days from discharge if the tests are negative.

\section{Conclusions}

There is an increased susceptibility of pregnant women to catch COVID-19, which may lead to morbidity and mortality not only during pregnancy but also postpartum. Till now, there is no sufficient evidence suggesting vertical transmission of SARS-COV-2, and therefore, vaginal delivery is not contraindicated. However, it is obligatory to take the necessary precautions for both the mother and the obstetricians. If there is a confirmed case of SARS-COV-2, all precautions for air transmission must be taken by all healthcare workers. No breastfeeding and total isolation of mother and neonate until complete recovery from SARSCOV-2 is confirmed. The fetal complications of COVID-19 that have been recorded so far, but preterm birth may be the most common fetal complication.

\section{Disclosure statement}

The authors state that there are no conflicts of interest.

\section{References}

1. Park SE (2020) Epidemiology, virology, and clinical features of severe acute respiratory syndrome-coronavirus-2 (SARS-CoV-2; Coronavirus Disease-19). Clin Exp Pediatr 63: 119-124.

2. Kourtis AP, Read JS, Jamieson DJ (2014) Pregnancy and infection. New Eng J Med 370: 2211-2218.

3. Zhao X, Jiang $Y$, Zhao Y, et al. (2020) Analysis of the susceptibility to COVID-19 in pregnancy and recommendations on potential drug screening. Eur J Clinl Microbiol Infect Dis 39: 1209-1220.

4. Collin J, Byström E, Carnahan A, et al. (2020) Pregnant and postpartum women with SARS-CoV-2 infection in intensive care in Sweden. Acta Obstet Gynecol Scand.

5. Karimi-Zarchi M, Neamatzadeh H, Dastgheib SA, et al. (2020) Vertical transmission of coronavirus disease 19 (COVID-19) from infected pregnant mothers to neonates: A review. Fetal Pediat Pathol 39: 246-250.

6. Asadi L, Tabatabaei RS, Safinejad H, et al. (2020) New Corona Virus (COVID-19) management in pregnancy and childbirth. Archives Clin Infect Dis 15(COVID-19): e102938.

7. Schwartz DA, Graham AL (2020) Potential maternal and infant outcomes from (Wuhan) coronavirus 2019-nCoV infecting pregnant women: Lessons from SARS, MERS, and other human coronavirus infections. Viruses 12: 194.

8. Dashraath P, Jeslyn WJ, Karen LM, et al. (2020) Coronavirus disease 2019 (COVID-19) pandemic and pregnancy. Am J Obstet Gynecol 222: 521-531.

9. Maxwell C, McGeer A, Tai KF, et al. (2009) Management guidelines for obstetric patients and neonates born to mothers with suspected or probable severe acute respiratory syndrome (SARS). J Obstet Gynaecol Can 31: 358-364.

10. Yu N, Li W, Kang Q, et al. (2020) Clinical features and obstetric and neonatal outcomes of pregnant patients with COVID-19 in Wuhan, China: A retrospective, single-centre, descriptive study. Lancet Infect Dis 20: 559-564.

11. Di Mascio D, Khalil A, Saccone G, et al. (2020) Outcome of Coronavirus spectrum infections (SARS, MERS, COVID 1-19) during pregnancy: A systematic review and meta-analysis. Am J Obstet Gynecol MFM 2: 100107.

12. Poon LC, Yang H, Kapur A, et al. (2020) Global interim guidance on coronavirus disease 2019 (COVID-19) during pregnancy and puerperium from FIGO and allied partners: Information for healthcare professionals. Int J Gynecol Obstet 149: 273-286.

13. Rasmussen SA, Smulian JC, Lednicky JA, et al. (2020) Coronavirus disease 2019 (COVID-19) and pregnancy: What obstetricians need to know. Am J Obstet Gynecol 222: 415-426.

14. Rollins NC, Bhandari N, Hajeebhoy N, et al. (2016) Why invest, and what it will take to improve breastfeeding practices? Lancet 387: 491-504.

15. Favre G, Pomar L, Qi X, et al. Guidelines for pregnant women with suspected SARS-CoV-2 infection. Lancet Infect Dis 20: 652-653.

16. Royal College of Obstetricians \& Gynecologists (2020) Coronavirus (COVID-19) infection in pregnancy. Information for healthcare professionals. Version 2.

17. Poon LC, Yang H, Lee JC, et al. (2000) ISUOG Interim Guidance on 2019 novel coronavirus infection during pregnancy and puerperium: Information for healthcare professionals. Ultrasound Obstet Gynecol 55: 700-708. 
Citation: Abbas AM, Ali SS, Mohamed AO, et al. (2021) COVID-19 with Pregnancy: Obstetricians should be Alert for Postpartum Exacerbation. Annals Gynecol Obstet 5(1):138-141

18. https://www.cdc.gov/coronavirus/2019-ncov/hcp/inpatientobstetric-healthcare-guidance.html
19. Dabrowski GA (2007) Skin-to-skin contact: Giving birth back to mothers and babies. Nursing for women's health 11: 64-71.

DOI: $10.36959 / 468 / 481$ 